\title{
DISEÑO SÍSMICO BASADO EN DESEMPEÑO: TENDENCIAS Y RETOS*
}

\author{
Luis Esteva Maraboto ${ }^{(1)}$
}

\section{RESUMEN}

Se presenta una breve revisión de los objetivos y los planteamientos del diseño sísmico basado en criterios de desempeño. Se empieza describiendo algunos lineamientos propuestos en la literatura internacional para el desarrollo de documentos normativos. Tales lineamientos incluyen ideas sobre indicadores de desempeño y sobre criterios de aceptación para temblores asociados con diversos intervalos esperados de recurrencia en el sitio de interés. Se discute el problema de seleccionar los parámetros de control idóneos para fines de diseño en condiciones de la práctica, así como la determinación de valores óptimos de dichos parámetros con base en un análisis costo-beneficio referido al ciclo esperado de vida de cada construcción. Para ello se definen los conceptos de función de daño y función de confiabilidad, las que para una estructura específica, o diseñada de acuerdo con un cierto criterio, se expresan en términos de las intensidades sísmicas o de las respuestas esperadas de un sistema simple equivalente que resultan ante dichas intensidades. Se trata brevemente la transformación de los resultados de los estudios de optimización en criterios prácticos de diseño que conduzcan a sistemas cuyas propiedades mecánicas se aproximan lo más posible a sus valores óptimos.

\begin{abstract}
A brief review is presented of the objectives and formulation of performance-based seismic design criteria. The paper starts with a description of some guidelines proposed in the international literature for the development of normative documents. Such guidelines include ideas about the variables that can be adopted as adequate performance indicators, as well as about acceptance criteria for those indicators under the action of earthquakes associated with different return intervals. The problem of selecting the most adequate control parameters for design in practical conditions is discussed, as well as that of determining the optimum values of those parameters on the basis of cost-benefit studies under a life-cycle framework. For this purpose, the concepts of damage function and reliability function are defined. For a specific structure, or for one that has been designed in accordance with a given criterion, such functions are expressed in terms of ground motion intensities or of the corresponding responses of a simplified equivalent system. A brief discussion is presented about the transformation of the results of the optimization studies into practical design criteria that should lead to structural systems with mechanical properties as close as possible to their optimum values.
\end{abstract}

\footnotetext{
* Versión actualizada de la Conferencia Magistral presentada durante el XII Congreso Nacional de Ingeniería Sísmica, Morelia, Mich. (Nov, 1999). Se aceptan comentarios y/o discusiones al artículo hasta cinco meses después de su publicación.
}

(1) Investigador, Instituto de Ingeniería, UNAM, e-mail: lesm@pumas.iingen.unam.mx. 


\section{INTRODUCCIÓN}

Los objetivos del diseño sísmico de obras de ingeniería son bien conocidos: se pueden resumir en el logro de un equilibrio adecuado entre los costos de construcción y de mantenimiento y los riesgos aceptables de daños o fallas durante la vida útil de cada obra. Esto implica, por una parte, mantener a un nivel suficientemente bajo el riesgo de colapso ante temblores de las mayores intensidades probables en el sitio de la construcción, de manera de ofrecer una protección adecuada a la integridad física de sus ocupantes y de salvaguardar sus bienes y, por otra, controlar las pérdidas económicas asociadas con posibles daños materiales, con costos de reparación y con la interrupción de las funciones de dicha construcción o de las actividades que en ella deban desarrollarse. Para la práctica de la Ingeniería Sísmica, estos objetivos generales conducen a varias metas específicas: se trata de garantizar la seguridad contra colapso ante temblores con intensidades asociadas a intervalos de recurrencia muy largos, de limitar los daños estructurales ante temblores de intensidades que correspondan a intervalos de recurrencia moderados, y de evitar los daños materiales, tanto en miembros estructurales como en elementos no estructurales, ante intensidades sísmicas que puedan presentarse con frecuencia en el sitio de interés. Los criterios que se adopten para definir las acciones de diseño, o los correspondientes intervalos de recurrencia, las variables de control y los márgenes de seguridad aceptables, deberán lograr el equilibrio entre costos de construcción, de mantenimiento y de daños que se menciona al inicio de este párrafo.

Para una intensidad sísmica dada, el desempeño esperado de una construcción puede describirse mediante indicadores cualitativos o semi-cuantitativos de su comportamiento o en términos de las características probables de su respuesta dinámica: amplitudes máximas y números de ciclos. Entre los indicadores del primer grupo se encuentran, por ejemplo, los niveles de agrietamiento de elementos estructurales y no estructurales, la seguridad de muebles, equipo y diversos contenidos ante volteo, o el nivel de incomodidad de los ocupantes ante los movimientos de los pisos. También se encuentran otros, más difíciles de evaluar, como las probabilidades de alcanzar diversos niveles de daño, incluyendo posibles modos de falla total o parcial. Todos estos indicadores muestran en general una fuerte correlación estadística con las variables de la respuesta dinámica del sistema que se tratan de controlar mediante el diseño sísmico: deformaciones de entrepiso, demandas de comportamiento por encima de los niveles de respuesta lineal, o aceleraciones locales, entre otras; por ello, resulta conveniente expresar los requisitos de diseño sísmico directamente en términos de estas últimas variables. Los requisitos de resistencia lateral, que han jugado un papel preponderante en los criterios de diseño sísmico convencionales, adoptan en este marco de referencia una función indirecta, que es la de controlar en los miembros estructurales críticos las amplitudes y los números de ciclos de comportamiento no lineal, y por ende la acumulación de daño. Se reconoce explícitamente en este planteamiento que el evento de que un sistema con una resistencia lateral dada falle ante un sismo no es una consecuencia directa de la insuficiencia de dicha resistencia, sino de la posible falta de capacidad del sistema para desarrollar las deformaciones demandadas por la acción sísmica, las cuales pueden o no depender de la resistencia lateral en cuestión. Para un sistema caracterizado por una forma de comportamiento ante ciclos alternantes de deformaciones en el intervalo de comportamiento no lineal, la importancia de la resistencia lateral reside en su influencia determinante sobre la capacidad de deformación. 
El párrafo anterior se refiere a condiciones de desempeño que están controladas por indicadores de deformación. Sin embargo, pueden identificarse también condiciones que se controlan directamente con resistencias estructurales: un ejemplo es la resistencia de la cimentación para evitar el volteo general del sistema antes de que se agote su capacidad de deformación lateral; otro, la resistencia en tensión diagonal que debe tener una viga o una columna para que puedan desarrollarse en sus extremos rotaciones dúctiles por flexión.

Como se verá en una sección posterior de este trabajo, en términos estrictos el problema de determinar las características requeridas del sistema estructural que conduzca al equilibrio óptimo entre costos de construcción, de mantenimiento y de daños esperados bajo condiciones de riesgo sísmico debe plantearse en el marco del ciclo de vida, que incluye la descripción en forma probabilista de las características de las excitaciones sísmicas significativas que puedan afectar al sistema durante su vida útil esperada. En condiciones prácticas, una forma de lograr diseños que se aproximen suficientemente a la solución óptima consiste en establecer las condiciones requeridas de desempeño (es decir, las condiciones de diseño y los criterios de aceptación) para un conjunto de intensidades sísmicas, cada una asociada a un intervalo de recurrencia especificado. Para tales fines, tanto las intensidades y las condiciones de diseño propuestas como los criterios de aceptación pertinentes deberán deducirse a partir de estudios que incluyan tanto planteamientos formales de optimización como calibraciones con los criterios previos y con la experiencia sobre comportamiento observado en eventos anteriores. Al estimar las respuestas sísmicas esperadas con el objeto de determinar indicadores de desempeño ante temblores específicos, deben tomarse en cuenta los valores probables de las propiedades mecánicas de cada sistema, en vez de considerar los valores nominales más desfavorables (o característicos) que se suponen al aplicar los requisitos de diseño.

De acuerdo con los criterios convencionales de diseño sísmico, la determinación de las resistencias requeridas de los miembros estructurales se basa en general en análisis de respuesta lineal para espectros reducidos por comportamiento no lineal; es decir, salvo casos especiales, bastan las herramientas del análisis dinámico lineal. En cambio, para aplicar criterios basados explícitamente en niveles de desempeño será necesario estimar desplazamientos y deformaciones de sistemas no lineales de múltiples grados de libertad. Además de las reducciones de la rigidez que acompañan al crecimiento de las deformaciones, el comportamiento no lineal de los sistemas estructurales típicos incluye la influencia del daño acumulado, que se manifiesta como deterioro de las rigideces y de las resistencias como función de la historia de deformaciones globales y locales. Durante los últimos años se han logrado grandes avances en el desarrollo de sistemas de computadora capaces de calcular las historias de las respuestas sísmicas de sistemas no lineales de múltiples grados de libertad con diversos modelos de leyes constitutivas para representar el proceso de acumulación de daño. Sin embargo, el esfuerzo que se requeriría para aplicar tales sistemas a la estimación de respuestas estructurales en la práctica de diseño es aún excesivo para la mayor parte de los casos, particularmente si se reconocen las limitaciones de nuestro conocimiento sobre el comportamiento de sistemas reales sujetos a historias de deformaciones cíclicas tan irregulares como las causadas por temblores. De aquí que uno de los retos importantes para el desarrollo de criterios de diseño sísmico basados en desempeño sea el de desarrollar y calibrar métodos simples, eficientes y confiables para estimar amplitudes máximas de las respuestas sísmicas de sistemas estructurales complejos de comportamiento no lineal. 
En lo que sigue se presentan en forma somera algunos conceptos fundamentales contenidos en varios documentos recientes sobre recomendaciones y lineamientos para establecer criterios de diseño sísmico basados en desempeño. Luego se discuten los criterios para describir el desempeño y los indicadores para expresarlo en forma cuantitativa y se presentan algunas ideas sobre los métodos y modelos simplificados para estimar las respuestas sísmicas de sistemas estructurales no lineales de múltiples grados de libertad. Se continúa con una discusión sobre los formatos para diseño en condiciones típicas de la práctica y se plantean los estudios de confiabilidad, riesgo, optimización y calibración que conviene llevar a cabo para fundamentar los criterios de diseño que se propongan. El trabajo termina con algunos comentarios sobre las perspectivas de desarrollo y los retos involucrados.

\section{RECOMENDACIONES PRELIMINARES}

Entre los ingenieros mexicanos, la baja eficacia de las normas de diseño sísmico vigentes para controlar los daños causados por temblores de intensidad baja y moderada había sido reconocida a partir de las observaciones sobre los daños causados en la ciudad de México por varios eventos de tales características ocurridos durante los años transcurridos entre los eventos destructivos de 1957 y 1985. Entre los ingenieros norteamericanos, se hizo notoria una preocupación similar a raíz del sismo de Loma Prieta en 1989; dicha preocupación se exacerbó a consecuencia de los severos daños causados por el temblor de Northridge en 1994. El análisis de los daños observados en estos eventos hizo concluir que, si bien las normas de diseño y construcción de estructuras resistentes a sismos habían cumplido razonablemente con su función de evitar fallas catastróficas y proteger vidas, su contribución al control de daños y pérdidas económicas distaba de ser satisfactoria.

En 1992, la Asociación de Ingenieros Estructurales de California (SEAOC, por sus siglas en inglés), en reconocimiento de "la necesidad de una nueva generación de procedimientos de diseño", estableció el Comité VISION 2000, con la misión de "mirar al futuro y desarrollar un marco de referencia para procedimientos que condujesen a estructuras de desempeño sísmico predecible”. Las conclusiones de ese comité se presentaron en dos volúmenes (SEAOC, 1995), que incluían un conjunto de recomendaciones preliminares y un marco conceptual para diseño por desempeño, además de un análisis preliminar de los daños causados por el temblor de Northridge y un conjunto de sugerencias para el proceso de incorporar las recomendaciones preliminares en el desarrollo de documentos normativos con el enfoque propuesto. Las recomendaciones preliminares incluyen la definición de varios niveles de desempeño y de varios niveles de las excitaciones sísmicas de diseño, estos últimos expresados en términos de su probabilidad de ocurrencia. La definición de los niveles de desempeño se expresa en términos cualitativos, comprensibles para el público general, y se complementa con los indicadores de tipo técnico que deberán usarse para el diseño y la evaluación en ingeniería.

Los niveles de desempeño se expresan en términos de los efectos de un sismo sobre la capacidad de la construcción para realizar sus funciones. Tales niveles se designan como plenamente operacional, operacional, seguridad de vidas y cercano al colapso. Los dos primeros se refieren a las condiciones de funcionamiento posteriores al sismo y el tercero implica la existencia de un amplio margen de seguridad con respecto al colapso. El significado del cuarto nivel es obvio. Para edificios ordinarios, a estos niveles de desempeño se hacen corresponder valores máximos de la distorsión 
global o local (desplazamiento relativo entre dos niveles, dividido entre la distancia vertical) iguales respectivamente a $0.002,0.005,0.015$ y 0.025 .

Los niveles de las excitaciones sísmicas de diseño se describen en términos cualitativos como frecuente, ocasional, rara y muy rara. A estos niveles corresponden respectivamente las probabilidades de excedencia de 50 por ciento en 30 años, 50 por ciento en 50 años, 10 por ciento en 50 años y 10 por ciento en 100 años o, en forma equivalente, intervalos de recurrencia de 43, 72, 475 y 970 años.

Los objetivos de desempeño establecen, para cada tipo de estructura, según su importancia y el nivel de riesgo tolerable, el nivel de desempeño que debe lograrse para cada nivel de excitación sísmica. Por ejemplo, para construcciones ordinarias, el documento en estudio recomienda las metas de niveles de desempeño que se resumen en la siguiente tabla:

Tabla 1. Metas de niveles de desempeño para edificios ordinarios

\begin{tabular}{|l|l|l|}
\hline $\begin{array}{c}\text { Nivel de } \\
\text { Excitación sísmica }\end{array}$ & $\begin{array}{l}\text { Intervalo de } \\
\text { Recurrencia }\end{array}$ & $\begin{array}{l}\text { Nivel mínimo de } \\
\text { desempeño }\end{array}$ \\
\hline \hline Frecuente & 43 años & $\begin{array}{l}\text { Plenamente } \\
\text { operacional }\end{array}$ \\
\hline Ocasional & 72 & Operacional \\
\hline Rara & 475 & Protección de vidas \\
\hline Muy rara & 970 & Cercano al colapso \\
\hline
\end{tabular}

Una mirada a esta tabla muestra que los intervalos de recurrencia propuestos para los niveles de desempeño operacional y plenamente operacional son significativamente mayores que los que se han logrado para un gran número de construcciones en la ciudad de México. No es posible comparar directamente los intervalos de recurrencia correspondientes a los niveles de desempeño de protección de vidas y de cercanía al colapso, ya que no se cuenta con estudios sistemáticos sobre los márgenes de seguridad que ofrecen las normas técnicas vigentes para diseño sísmico con respecto a los correspondientes niveles de desempeño cuando se presentan las excitaciones sísmicas de los niveles especificados. Por ejemplo, en el caso particular de la zona de terreno blando de la ciudad de México, los espectros establecidos en las Normas Técnicas Complementarias del Reglamento de Construcciones del Distrito Federal para el nivel de protección de vidas corresponden a un intervalo de recurrencia de aproximadamente 125 años, que es muy inferior al de 475 años, sugerido por el Comité VISION 2000. Estos márgenes de seguridad dependen, entre otros conceptos, de las relaciones implícitas entre los valores más probables de las cargas gravitacionales y de las propiedades mecánicas de los miembros estructurales y los correspondientes valores nominales de estas variables que se suponen en la práctica del diseño. Más adelante se presentan algunos estudios preliminares para calibrar los niveles de confiabilidad implícitos en las normas citadas con respecto a las metas de niveles de desempeño de la Tabla 1. También se discute el problema de establecer valores óptimos de los parámetros de diseño sísmico basado en criterios de desempeño. 


\section{INDICADORES DE DESEMPEÑO}

En el marco de su ciclo esperado de vida, el desempeño de una obra de ingeniería puede describirse cuantitativamente por el valor esperado de todos los beneficios y costos que puedan presentarse en el futuro, transformados a su valor presente para el instante en que se termina su construcción y empieza a desempeñar las funciones para las que fue proyectada. El valor mencionado resulta de sumar los costos iniciales de construcción con los valores actualizados de los beneficios futuros esperados, así como de las posibles erogaciones por concepto de mantenimiento o las que puedan resultar a consecuencia de las acciones que afecten a la obra durante su vida útil esperada. Entre estas se encuentran los daños materiales, tanto en la estructura como en sus contenidos y acabados, y las pérdidas por interrupción de operaciones, así como otras que no pueden expresarse en términos económicos, como los daños a la salud o a la integridad de personas.

Una fracción importante de los daños en miembros estructurales y en elementos no estructurales se asocia directamente a agrietamientos o fallas causados por deformaciones excesivas. En marcos de concreto reforzado diseñados con criterios que eviten que se presente en un miembro cualquiera la falla por tensión diagonal antes de que ocurra la falla en un modo dúctil, tal como la fluencia del refuerzo en secciones críticas por momento flexionante, el agrietamiento se inicia en general en los extremos de los miembros, y su intensidad se relaciona estrechamente con la curvatura local. En marcos continuos de acero estructural, los mayores daños en miembros individuales suelen también estar asociados a curvaturas excesivas en los extremos, aunque en este caso pueden manifestarse a través del pandeo local de los patines de compresión o del pandeo local del alma causado por esfuerzos cortantes en su plano; son posibles también las fallas de tipo frágil en las conexiones entre miembros de flexión, sean juntas soldadas, remachadas o atornilladas. En todos los casos citados en este párrafo, los daños locales dependen de los números de ciclos de diversas amplitudes de deformación, y no exclusivamente de los valores máximos de dichas amplitudes.

En sistemas estructurales cuyas capacidades para resistir acciones laterales dependan fundamentalmente de elementos de tipo diafragma, los daños se correlacionan principalmente con las distorsiones laterales; en edificios típicos, dichas distorsiones se obtienen como los cocientes de las deformaciones relativas de entrepiso entre las alturas correspondientes. También en los casos de marcos estructurales continuos, como los mencionados en el párrafo anterior, para fines de evaluar el desempeño puede optarse por criterios aproximados, referidos a las amplitudes de las distorsiones laterales de entrepiso, como una forma simplificada de tomar en cuenta las deformaciones locales en las regiones críticas de los miembros estructurales.

Diversos elementos no estructurales, tales como muros de relleno, revestimientos y acabados, puertas y ventanas, son sensibles también a las distorsiones de entrepiso; otros, como instalaciones y elementos colgantes, anaqueles y diversos tipos de equipo, son sensibles a las aceleraciones locales de los puntos en que se conectan al sistema estructural. Lo mismo ocurre con los tableros de muro que no se liguen a dicho sistema por medio de anclajes en sus bases o de cualquier otro tipo de elementos que impidan su volteo. 


\section{CAPACIDADES DE DEFORMACIÓN}

Los valores de los indicadores cuantitativos de desempeño relativos a los modos de daño o falla que dependen de deformaciones locales o globales pueden calcularse para un temblor de una intensidad dada en términos de los cocientes de los valores esperados de las deformaciones máximas entre las correspondientes capacidades de deformación. En general, tales capacidades dependen de la configuración del sistema cuando se presenta la máxima amplitud de la respuesta de interés. Por ejemplo, el indicador de desempeño relativo al comportamiento de un entrepiso dado será función del cociente de la amplitud máxima de la distorsión lateral de dicho entrepiso durante la respuesta al sismo considerado entre la capacidad de deformación de dicho entrepiso. Aun si se no se considera explícitamente la influencia de los números de ciclos de deformación de distintas amplitudes, la curva carga-deformación del entrepiso de interés dependerá de las historias de deformación de las vigas y columnas que pertenecen a dicho entrepiso, así como de las correspondientes a los miembros estructurales de los entrepisos vecinos. Implicaría excesivas dificultades prácticas el tratar de determinar la configuración del sistema en cada instante en que se produce el máximo de alguna deformación de interés, con el fin de calcular la correspondiente capacidad de deformación. Por ello se ha desarrollado un criterio aproximado, basado en suponer que dicha configuración coincide con la determinada a partir del análisis de "empuje lateral" ( "pushover analysis", según la literatura en inglés sobre el tema). Dicho análisis consiste en determinar la respuesta del sistema de interés ante un sistema de cargas laterales que crecen lentamente en forma proporcional, sin causar efectos dinámicos (Qi y Moehle, 1991). Los resultados de dicho análisis se expresan en términos de una curva de comportamiento global y de una forma de variación de las configuraciones de desplazamientos laterales del sistema con respecto a la amplitud de la respuesta. La primera se representa como la relación entre la fuerza cortante en la base $(V)$ y el desplazamiento relativo del extremo superior con respecto a la base $\left(u_{N}\right)$; la segunda, mediante un conjunto de configuraciones que se mantienen proporcionales en el caso de sistemas lineales y que varían en función de la amplitud de $u_{N}$ en el caso de sistemas no lineales. Se han propuesto diversos criterios para definir la forma del sistema de cargas laterales; se incluyen entre ellos los que sugieren tomar la forma del vector de fuerzas de inercia asociado al modo fundamental de vibración o a una superposición de las contribuciones de los modos que más contribuyen a la respuesta. También se ha sugerido aplicar como excitación una aceleración

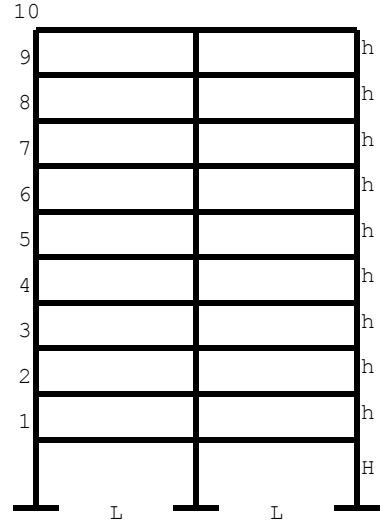

Elevación

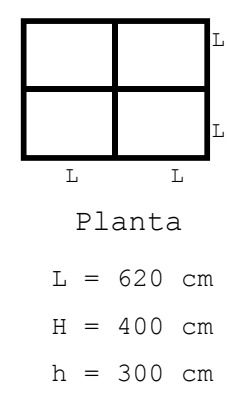

Fig. 1. Edificio de 10 niveles

$=400 \mathrm{~cm}$

$300 \mathrm{~cm}$

\begin{tabular}{|c|c|r|}
\hline Elemento & Nivel & Sección transversal \\
\hline & & HxB (cm) \\
\hline Columnas & $1-4$ & $80 \times 80$ \\
\hline & $5-7$ & $65 \times 65$ \\
\hline Trabes & $8-10$ & $50 \times 50$ \\
\hline & $1-5$ & $65 \times 30$ \\
\hline & $6-8$ & $60 \times 30$ \\
\hline
\end{tabular}


lentamente creciente en la base del sistema. (En este caso, la forma del vector de fuerzas de inercia no se mantiene constante al crecer el desplazamiento relativo del extremo superior.) Como ejemplo, en la Fig.1 se presenta un marco de diez niveles estudiado por Esteva et al (2000) en el proceso de desarrollar procedimientos aproximados para estimar índices de desempeño en sistemas no lineales de múltiples grados de libertad. La Fig.2 (tomada de Esteva et al, 2000) presenta la respuesta del sistema a una aceleración de la base lentamente creciente en forma lineal con el tiempo. Dicha respuesta se representa mediante una curva $V-u_{N}$ y un conjunto de curvas que representan las configuraciones de respuesta lateral que corresponden a diversos valores de la última variable. Los saltos en la curva $V-u_{N}$ corresponden a los instantes en que se alcanzan las capacidades de deformación en las secciones críticas de algunos miembros estructurales. Las vibraciones de corta duración que se observan son consecuencia de los cambios bruscos de propiedades mecánicas asociados a las fallas locales.

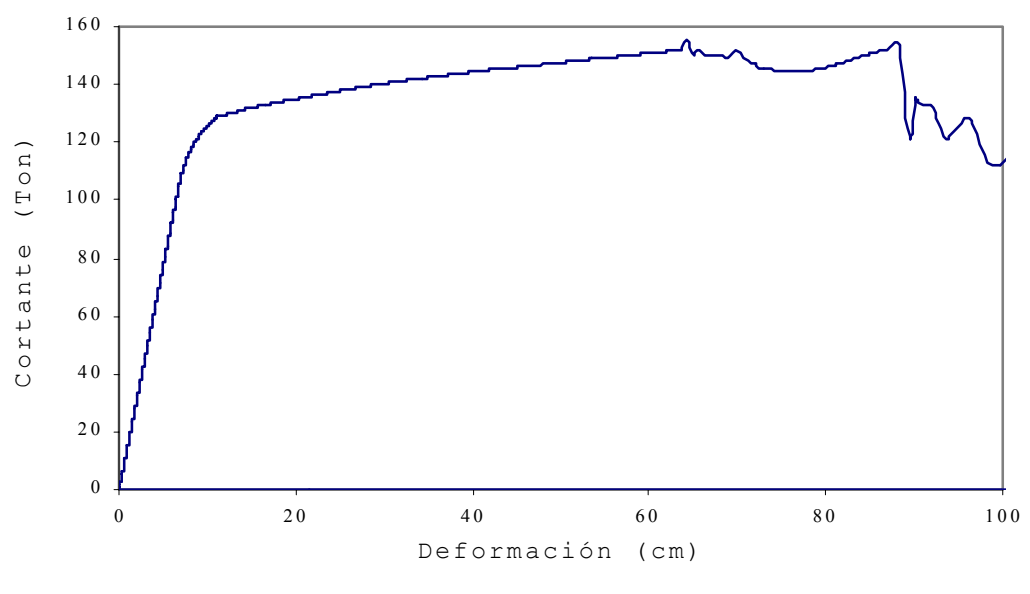

a) Curva de cortante en la base vs desplazamiento en azotea

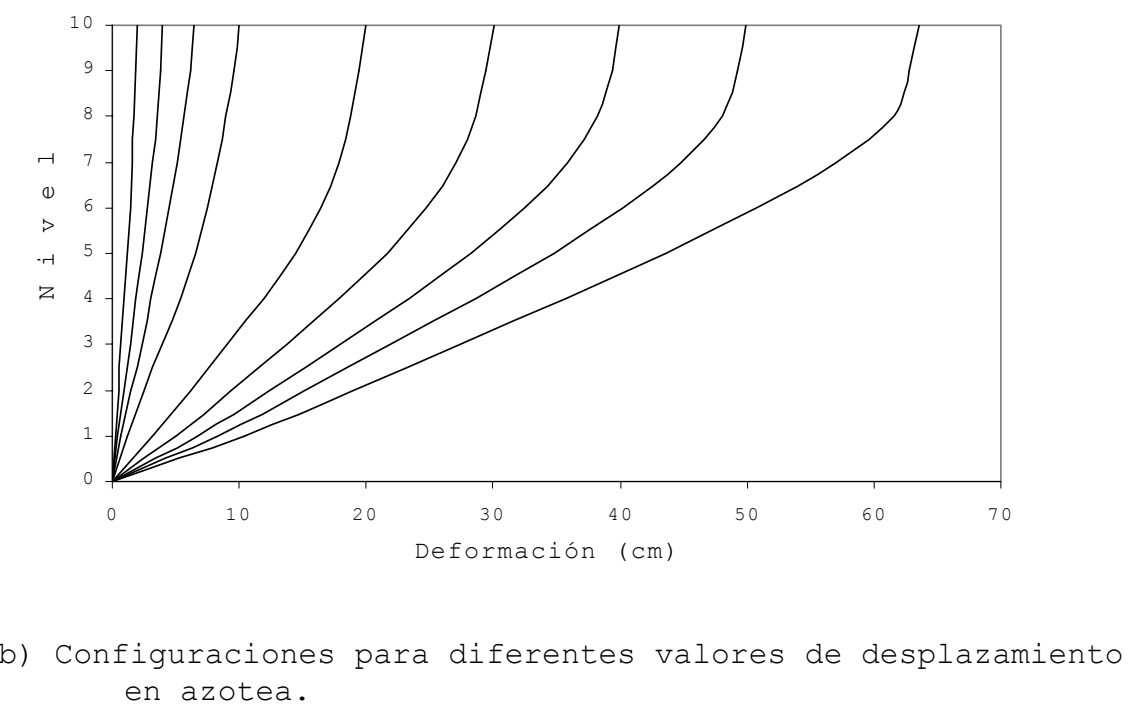

Fig. 2. Resultados del análisis del empuje lateral (push-over) 


\section{ESTIMACIÓN DE RESPUESTAS SÍSMICAS}

Para que las ventajas potenciales de los métodos de diseño sísmico basados en desplazamientos puedan aprovecharse es necesario contar con procedimientos de análisis que permitan obtener estimaciones precisas y confiables de las variables de respuesta más estrechamente relacionadas con los indicadores de desempeño; estas variables son las deformaciones locales y las distorsiones laterales en distintos puntos en la altura del sistema. Entre los procedimientos que satisfacen los requisitos citados se encuentran los métodos paso-a-paso para análisis de respuestas dinámicas. El esfuerzo de cálculo que se requiere en general para aplicar estos métodos en condiciones de la práctica del diseño estructural es excesivo, por lo que se ha propuesto la alternativa de estimar el desplazamiento relativo máximo del extremo superior del sistema de interés con respecto a su base aplicando el método de análisis paso a paso a un sistema simplificado equivalente, de un grado de libertad, con una curva fuerza-deformación igual a la curva $V-u_{N}$ determinada por el método del empuje lateral descrito arriba. En tal caso, los máximos de las deformaciones locales y de entrepiso se estiman suponiendo que cuando dichos máximos ocurren es válida la configuración de respuesta lateral que se obtiene mediante el método del empuje lateral para un desplazamiento relativo del extremo superior igual al estimado con el sistema simplificado equivalente.

De acuerdo con lo anterior, las respuestas (desplazamientos, deformaciones, aceleraciones locales) estimadas por medio del sistema simplificado equivalente son únicamente valores aproximados, cuya relación con los valores determinados mediante modelos más precisos puede expresarse en términos de un factor correctivo incierto, descrito en forma probabilista por medio de su valor esperado y su coeficiente de variación (Esteva et al, 2001).

Para aplicar el sistema simplificado equivalente a la práctica del diseño sísmico se parte de una primera aproximación a las propiedades mecánicas de los miembros estructurales y se determinan la curva $V-u_{N}$ y las configuraciones de respuesta lateral del sistema como función de $u_{N}$, por medio de un análisis de empuje lateral. Si se considera conveniente, se ajusta a la curva $V-u_{N}$ una función bilineal, como la mostrada en la Fig.2a. A partir de esta función se define la correspondiente al sistema simplificado. Para ello es necesario incorporar dos factores correctivos (Esteva et al, 2000); uno toma en cuenta el factor de participación dinámica de la configuración supuesta para sustituir el sistema de múltiples grados de libertad por su sistema equivalente, y el otro introduce un cambio de escala en el eje horizontal a fin de lograr la equivalencia entre los niveles de comportamiento no lineal de los sistemas detallado y equivalente. Aplicando estas transformaciones, es fácil estimar el valor máximo del desplazamiento relativo del extremo superior del sistema detallado $\left(u_{N}\right)$ a partir del que corresponde al sistema simplificado. Para estimar este último puede realizarse un análisis de respuesta dinámica no lineal o puede aprovecharse un espectro de diseño para sistemas bilineales. Dado el valor máximo de $u_{N}$, es posible estimar cualquier medida de deformación local, de entrepiso o global, por simple proporcionalidad a partir de la configuración de respuesta lateral determinada mediante el análisis de empuje lateral para dicho valor de $u_{N}$. En el Apéndice se presentan detalles de este procedimiento aproximado de análisis de respuesta sísmica.

El último paso consistirá en introducir un factor correctivo que toma en cuenta las incertidumbres asociadas con las simplificaciones que conducen al sistema equivalente: la adopción de una configuración de respuesta lateral que es función exclusivamente del desplazamiento máximo del 
extremo superior del sistema, y la adopción de leyes constitutivas que ignoran los efectos de degradación de rigideces y resistencias que se presentan en los modelos refinados del sistema como consecuencia de la ocurrencia de ciclos repetidos de deformaciones de gran amplitud. Una forma de incorporar estas incertidumbres en las estimaciones de las deformaciones de interés se ilustra en los párrafos que siguen, tomando como ejemplo las distorsiones de entrepiso de un marco de edificio.

Volviendo al marco plano de la Fig.1, supóngase la acción de un temblor definido por su intensidad $y$, la cual puede medirse, por ejemplo, por la ordenada del espectro lineal de respuesta de seudoaceleraciones, para un valor dado del coeficiente de amortiguamiento. Suponiendo que no se conocen las características detalladas del movimiento del terreno, no será posible conocer el valor del desplazamiento del sistema simplificado, y por tanto tampoco el del extremo superior del modelo detallado del sistema de interés. Supondremos que contamos con un conjunto de espectros de respuesta para sistemas bilineales como el sistema simplificado de interés. A partir de ellos será fácil determinar el valor esperado del máximo desplazamiento relativo $u_{N}$. En lo que sigue resultará práctico trabajar con la distorsión global $\psi$ del sistema, es decir, el cociente de $u_{N}$ entre la altura del marco. A la esperanza del máximo valor que pueda alcanzar $\psi$ durante el temblor de interés se le designará por $\bar{\psi}$. A los valores de $\psi$ y $\bar{\psi}$ estimados por medio del sistema equivalente se les designará por $\psi_{0}$ y $\bar{\psi}_{0}$, respectivamente. De manera semejante, se introducen los símbolos $\psi_{i}, \psi_{0 i}, \bar{\psi}_{i}$ y $\bar{\psi}_{0 i}$, para designar a la distorsión máxima alcanzada por el $i$-ésimo entrepiso, su valor estimado por medio del sistema equivalente y los valores esperados de ambas variables, respectivamente. El cociente $\psi_{i} / \bar{\psi}_{0 i}$ es una variable aleatoria, que se designa en lo que sigue por $\rho_{i}$; su media $\bar{\rho}_{i}$ y su coeficiente de variación $V_{\rho i}$ pueden estimarse a partir de bases de información que se establezcan a partir de los resultados de estudios detallados de respuesta para sistemas similares al de interés. El establecimiento de estas bases de información es uno de los retos inmediatos para la aplicación de sistemas simplificados a la estimación de respuestas máximas de sistemas no lineales de múltiples grados de libertad.

Dado el valor de $\bar{\psi}_{0 i}$ para un entrepiso de un sistema como el de la Figura 1, el valor de $\psi_{i}$ que deba suponerse para diseño podrá estimarse mediante la siguiente ecuación:

$$
\psi_{i}^{\star}=\bar{\psi}_{0 i} \bar{\rho}_{i}\left(1+\alpha V_{\rho i}\right)
$$

Aquí, $\alpha$ es un parámetro que controla la probabilidad de que el valor real de $\psi_{i}$ sea mayor que su valor calculado según la Ec.1.

\section{FUNCIONES DE DAÑO}

Si se conociera en detalle la historia de respuestas de un sistema como el de la Fig.1 ante un movimiento sísmico, sería posible determinar el comportamiento del sistema y de sus contenidos y transformar dicha información en un monto global de daños, el cual resultaría de sumar los montos de daños asociados con los diversos modos de daño o falla del sistema y de sus contenidos. Si las características de la excitación sísmica están definidos únicamente por medio de su intensidad $y$, el monto global de daños resultará expresado en términos de su valor esperado, designado por $\delta(y) C_{0}$, en 
donde $C_{0}$ es el costo de construcción del sistema, el cual depende de los parámetros de diseño, $\underline{\kappa}$. Para un marco como el de la Fig.1, $\delta(y)$ puede calcularse como la suma de las contribuciones de distintos segmentos (entrepisos) del sistema, de acuerdo con lo que sigue:

$$
\delta(y)=\sum_{i} r_{c i} \bar{g}\left(\psi_{i}\right)
$$

En esta ecuación, $r_{c i}=C_{0 i} / C_{0} ; C_{0 i}$ es el costo inicial del $i$-ésimo segmento del sistema; $g\left(\psi_{i}\right)$ es una función del valor aleatorio $\psi_{i}$ de la distorsión local, y $\bar{g}\left(\psi_{i}\right)$ es su valor esperado. Los costos iniciales $C_{0}$ y $C_{0 i}$ son funciones del vector $\underline{\kappa}$. De este vector depende también la densidad conjunta de probabilidades de las distorsiones locales $\psi_{i}$. Las funciones $g\left(\psi_{i}\right)$ pueden estimarse a partir de información empírica (Reyes, 1999; Esteva et al, 2001). La influencia de la incertidumbre asociada con el valor máximo de $\psi_{i}$ para una intensidad dada, $y$, pueden tomarse en cuenta mediante la estimación bipuntual propuesta por Rosenblueth (1975):

$$
\bar{g}\left(\psi_{i}\right)=\frac{1}{2}\left[g_{i}\left\{\bar{\psi}_{0 i}(y) \bar{\rho}_{i}\left(1+V_{\rho i}\right)\right\}+g_{i}\left\{\bar{\psi}_{0 i}(y) \bar{\rho}_{i}\left(1-V_{\rho i}\right)\right\}\right]
$$

La aplicación de las Ecs. 2 y 3 al marco mostrado en la Fig.1 condujo a la función de daño mostrada en la Fig.3 (Esteva et al, 2001); en ella, la intensidad y está expresada por medio de la ordenada $S_{a}$ del espectro de seudoaceleraciones para un periodo natural igual al del sistema y amortiguamiento igual a 0.05 del crítico. Para cada entrepiso, la función $\bar{g}\left(\psi_{i}\right)$ se tomó igual a 1-exp($\left.a \psi^{-m}\right)$, con $a=2375$ y $m=2$, obtenidos con base en la información presentada por Reyes (1999).

\section{ANÁLISIS DE OPTIMIZACIÓN PARA ESTABLECER PARÁMETROS PARA DISEÑO POR DESEMPEÑO}

En los párrafos que siguen se presenta un planteamiento formal para establecer las propiedades óptimas de un sistema estructural, con base en criterios que toman en cuenta el desempeño esperado del sistema ante los sismos de diversas intensidades que puedan afectarlo. Con ello se trata de mostrar diversos conceptos que deben tener en cuenta los responsables de formular criterios de diseño sísmico basados en desempeño. Sin embargo, el esfuerzo que se requiere para aplicar estos conceptos hace que su empleo para casos específicos únicamente pueda justificarse para sistemas de importancia excepcional. Más adelante se habla del problema de traducir los resultados de estudios de optimización en recomendaciones codificadas de aplicabilidad práctica.

Según se definió en la sección anterior, $\underline{\kappa}$ es un vector formado por los valores de los parámetros que determinan las propiedades mecánicas significativas de un sistema por diseñar. De acuerdo con una opción propuesta por Rosenblueth (1976), el análisis de optimización referido al ciclo esperado de vida que se presenta a continuación se basa en la adopción a priori de una estrategia de reparación y reconstrucción que establece que el sistema se repara o se reconstruye inmediatamente después de cada temblor que causa daños, empleando las mismas especificaciones del sistema original. $\mathrm{Si}$ las incertidumbres relativas a las propiedades del sistema son pequeñas en comparación con las que 
se asocian a las intensidades y a las características detalladas de los eventos sísmicos, los valores óptimos de los elementos de $\underline{\kappa}$ son aquellos que minimizan la siguiente función objetivo:

$$
U=C_{0}(\underline{\kappa})+\frac{D_{0}(\underline{\kappa})}{\eta}
$$

Aquí, $C_{0}(\underline{\kappa})$ es la función de costo inicial, $D_{0}$ es el costo esperado de daños y fallas por unidad de tiempo y $\eta$ es una tasa real de descuento (o interés). $D_{0}$ depende de la función de daño $\delta(y)$, definida con anterioridad, y de la actividad sísmica en el sitio de interés:

$$
D_{0}=-C_{0} \int \frac{d v_{Y}(y)}{d y} \delta(y) d y
$$

En esta ecuación, $v_{Y}(y)$ es la tasa anual de ocurrencia de temblores con intensidad mayor que $y$ en el sitio. De acuerdo con los párrafos anteriores, las propiedades del sistema que integran el vector $\underline{\kappa}$ son las variables independientes de la función $U$ por minimizar. Dichas propiedades son independientes de las excitaciones sísmicas que puedan ocurrir en el sitio. Sin embargo, las recomendaciones y normas típicas de diseño sísmico requieren expresarse en términos de valores aceptables de las respuestas estructurales que se presenten ante temblores asociados con periodos de recurrencia especificados. Por ello, conviene considerar dos planteamientos alternativos para definir los parámetros que integran el vector $\underline{\kappa}$ : uno lo hace en términos de las propiedades del sistema y otro por medio de valores aceptables de las respuestas para temblores de diseño especificados. De acuerdo con el primer planteamiento, el sistema se define por su periodo fundamental de vibración, $T$, su coeficiente de cortante en la base, $c_{y}$, para la deformación de fluencia correspondiente a la curva $V$ - $u_{N}$ de la Fig.2a, y las formas de variación de los valores locales de la resistencia y de la rigidez de entrepiso a lo largo de la altura del edificio. Estas variables, junto con los valores locales de la capacidad de deformación, determinan el valor de la distorsión lateral a la falla, $\psi_{F}=u_{N F} / H$, en donde $H$ es la altura del sistema y $u_{N F}$ es el valor último del desplazamiento relativo de su extremo superior con respecto a su base. En el estudio de casos específicos conviene agrupar estos parámetros como sigue, para fines de realizar el análisis de optimización: $\kappa_{1}=T, \kappa_{2}=c_{y}, \kappa_{3}=\psi_{F}$, y los vectores $\underline{\kappa}_{A} \mathrm{y} \underline{\kappa}_{B}$, que determinan respectivamente las formas de variación de las rigideces y de las resistencias laterales de entrepiso a lo largo de la altura del sistema. La traducción de los valores óptimos que resulten para estos parámetros para algunos sistemas típicos en conjuntos de temblores de diseño, variables de control y criterios de aceptación, constituye un reto importante para los responsables de establecer normas de diseño sísmico basadas en indicadores de desempeño.

Con fines de ilustración, se consideró el problema de determinar el valor óptimo del periodo fundamental de vibración de un marco similar al mostrado en la Fig.1, suponiendo la función de peligro sísmico $v_{Y}(y)$ que corresponde a un sitio en terreno blando en la ciudad de México. El coeficiente de actualización $\eta$ se tomó igual a 0.05. A fin de desacoplar este problema del correspondiente a la determinación del valor óptimo de $c_{y}$, se supuso que este último es suficientemente elevado como para hacer que la mayor contribución a $D_{0}$ según la Ec.4 provenga de los temblores frecuentes de intensidades bajas y moderadas. Además, se supuso que al variar el periodo natural de vibración las relaciones entre rigideces de los miembros estructurales se mantenían constantes, iguales a las del marco de la Fig.1. Para la función $C_{0}$ se adoptó una expresión propuesta por Vargas (1991): 


$$
C_{0} / C_{00}=1+q\left(T_{0}-T\right)^{n}
$$

En esta ecuación, $T_{0}$ es el periodo fundamental del sistema si se hubiera diseñado únicamente para resistir cargas gravitacionales, $C_{00}$ es el costo que correspondería a dicho diseño, y $q, n$ son parámetros empíricos. Para edificios ficticios con propiedades similares a las que se obtendrían en edificios de la ciudad de México si no se diseñaran ante temblores, Reyes (1999) obtuvo la relación $T_{0}$ $=0.11 N+0.56$, en donde $N$ es el número de pisos.

Con base en lo anterior, el valor óptimo de $T$ resultó igual a $1.15 \mathrm{~s}$. Para este periodo, el valor de $S_{a}$ que corresponde a un intervalo de recurrencia de diez años (es decir, $v_{Y}(y)=0.1$ ), determinado a partir de la curva de peligro sísmico para el sitio de interés, es igual a $0.140 \mathrm{~g}$. Las distorsiones laterales que resultan para esta excitación se muestran en la Fig.4, en donde el máximo ocurre en el cuarto entrepiso y vale 0.0027 . Este es el valor que debería recomendarse para el diseño para condiciones de servicio para un intervalo de recurrencia de diez años. Sin embargo, el amplio intervalo de variación que se observa en las distorsiones de entrepiso arroja dudas sobre la validez de un análisis de optimización como el presentado aquí, el cual caracteriza a las propiedades de rigidez lateral del sistema mediante un parámetro único, que es el valor del periodo fundamental. Es claro que el vector $\underline{\kappa}$ debería incluir uno o varios parámetros que describieran la variación de las rigideces de entrepiso en la altura del sistema. Una alternativa que merece estudiarse consistiría en tomar como variable independiente la cota superior a las distorsiones de entrepiso para un movimiento sísmico asociado a un intervalo de recurrencia especificado.

En los últimos párrafos se trató el problema de determinar los valores óptimos de las rigideces de entrepiso en forma desacoplada de las variables que describen las resistencias del sistema. En los casos reales, los valores óptimos de las variables de ambos grupos no son mutuamente independientes; es decir, deberían obtenerse mediante la solución a un sistema de ecuaciones simultáneas de optimización. La solución obtenida, sin embargo, puede tomarse como una aproximación inicial que puede servir, cuando menos, como punto de partida para una solución iterativa del sistema citado. Para fines prácticos, convendría determinar las condiciones requeridas para que dicha aproximación se encuentre suficientemente cerca de la solución óptima.

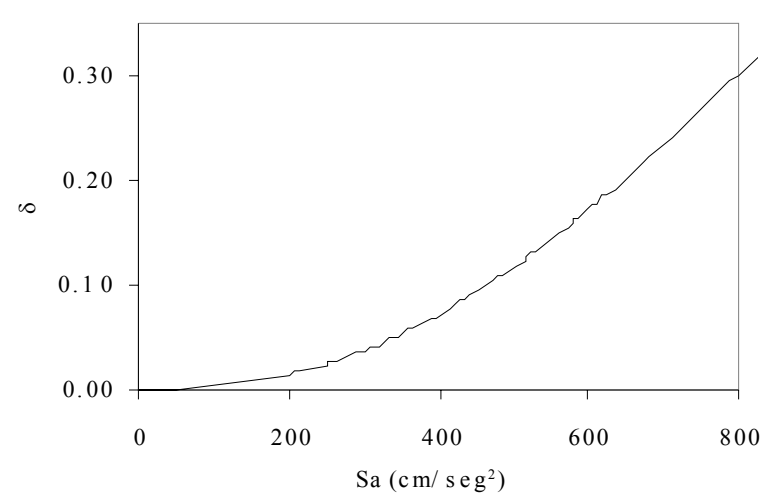

Fig. 3 Función de daño

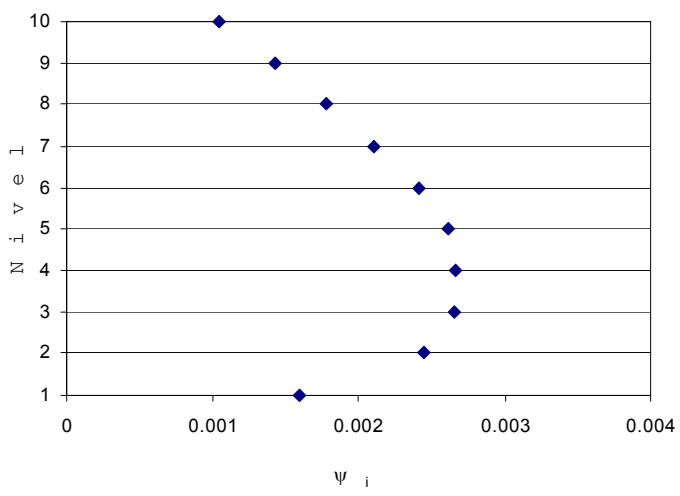

Fig. 4 Distorsiones de entrepiso para valor óptimo de periodo natural 


\section{FUNCIONES DE CONFIABILIDAD}

Por simplicidad, en la función de daño, $\delta(y)$, presentada en la Ec.2, y aplicada mediante la Ec.5 al problema de optimización descrito en la sección anterior, no se incorporó la contribución del posible evento de falla asociada al estado último de capacidad de deformación. Como se comentó, esta hipótesis es adecuada para estimar los costos esperados de daños asociados con intensidades sísmicas bajas, así como para la evaluación de costos esperados por unidad de tiempo, calculados según la Ec.5, cuando la aportación de los montos asociados con intensidades elevadas, pero poco frecuentes, a la integral de dicha ecuación es poco considerable en comparación con las aportaciones de las intensidades bajas, pero frecuentes. Este es el caso tratado arriba, cuando el interés se centra en determinar valores óptimos de las propiedades de rigidez del sistema. Para fines de determinar los valores óptimos de las propiedades de resistencia, que determinan, junto con las propiedades de ductilidad, las capacidades de deformación última del sistema, las consecuencias de la falla ante el estado límite último deben incluirse en forma explícita. En estas condiciones, $\delta(y)$ debe calcularse como sigue:

$$
\delta(y)=\delta_{S}(y)+p_{F}(y)\left[\delta_{F}-\delta_{S}(y)\right]
$$

Aquí, $p_{F}(y)$ es la probabilidad de que ocurra la falla ante el estado de capacidad última (colapso) para una intensidad igual a $y, \delta_{F} C_{0}$ es el monto de las consecuencias esperadas de dicho evento, en caso de ocurrir, y $\delta_{S}(y)$ es el costo esperado de daños para la intensidad $y$, para la condición de supervivencia del sistema. Este es el valor dado por el segundo miembro de la Ec.2.

La determinación de $p_{F}(y)$ para un sistema de múltiples grados de libertad y, por lo tanto, con múltiples modos de falla, implica estimar la probabilidad de que, para cualquiera de dichos modos de falla, la deformación demandada por la respuesta dinámica del sistema exceda de la correspondiente capacidad de deformación. En el caso de edificios con estructura constituida por marcos continuos, los modos de falla más probables consisten en el agotamiento de las capacidades de deformación de entrepiso. Esteva y Ruiz (1989) presentaron un procedimiento para estimar un índice de confiabilidad, $\beta$, semejante al propuesto inicialmente por Cornell (1969):

$$
\beta=-\frac{E[\ln z]}{\sigma[\ln z]}
$$

Aquí, $Z$ es el cociente de la demanda de deformación entre la correspondiente capacidad en el entrepiso en donde dicho cociente adquiere su máximo valor durante un temblor con una intensidad dada. $E[\cdot]$ y $\sigma[\cdot]$ denotan el valor esperado y la desviación estándar, respectivamente. Bajo la suposición de que $Z$ tiene distribución logarítmico-normal, $\beta$ puede ligarse con la probabilidad de falla para la intensidad en cuestión por medio de la relación $p_{F}(y)=\Phi(-\beta)$, en donde $\Phi(\cdot)$ es la función de distribución normal estándar.

En el artículo de Esteva y Ruiz (1989) el numerador y el denominador del segundo miembro de la Ec.8 se evalúan mediante simulación de Monte Carlo. Para este fin, se simula un conjunto de 
acelerogramas artificiales, en congruencia con un conjunto adecuado de suposiciones relativas a la evolución de las propiedades instantáneas de intensidad y contenido de frecuencias (varianza y densidad espectral, respectivamente). Las incertidumbres asociadas con las cargas gravitacionales que actúan sobre el sistema, así como las relacionadas con las propiedades mecánicas de sus miembros estructurales, se toman en cuenta también mediante simulación de Monte Carlo. Para ello se emplea la información disponible sobre las desviaciones estadísticas de los valores de dichas variables con respecto a sus valores nominales supuestos al diseñar. La capacidad de deformación de un entrepiso dado se estima, por ejemplo, como el producto de la deformación de fluencia de dicho entrepiso ante fuerza cortante por un factor de ductilidad con valor incierto. El valor esperado y el coeficiente de variación de dicho factor se estiman con base en sus valores nominales, de acuerdo con las suposiciones de diseño. Esteva et al (2000a) presentan algunos resultados sobre las ductilidades de entrepiso disponibles en marcos de concreto reforzado, calculadas a partir de las capacidades de deformación lateral local en vigas y columnas.

Esteva et al (2000a) presentan también algunos resultados sobre la forma de variación del índice $\beta$ de confiabilidad como función de la respuesta de un modelo simplificado del sistema de interés. El modelo simplificado es un sistema de un grado de libertad y comportamiento elastoplástico, con periodo natural igual al fundamental del sistema de interés, e igual coeficiente de amortiguamiento viscoso. Los periodos naturales de los sistemas considerados varían entre $0.5 \mathrm{~s}$ y $2.0 \mathrm{~s}$. Las excitaciones sísmicas fueron acelerogramas simulados de distintas intensidades, representativos de los que pueden presentarse en un sitio de terreno blando en el Valle de México (SCT). Entre los resultados del estudio se encuentran los presentados en la Fig.5 de este trabajo. Los valores mostrados en la parte superior de dicha figura corresponden a marcos con miembros estructurales cuyo comportamiento momentocurvatura puede representarse mediante gráficas histeréticas de tipo bilineal; los de la parte inferior corresponden a miembros estructurales cuyo comportamiento ante cargas cíclicas se caracteriza por degradación de rigideces, según el modelo de Takeda (Otani, 1974). En las abscisas se presentan valores de $Q$, que es la demanda de ductilidad del modelo simplificado del sistema ante un temblor de la intensidad que interesa. Puede observarse que el índice de seguridad varía en forma casi lineal con el logaritmo de la demanda de ductilidad en el sistema simplificado equivalente. Además, para el caso de funciones momento-curvatura de tipo bilineal la relación entre ambas variables no depende del número de pisos. Para los casos en que se tiene deterioro de rigideces en las funciones momento-curvatura, los índices de confiabilidad son en general menores que para los casos de comportamiento bilineal, para iguales valores de la demanda de ductilidad en el modelo simplificado. Esto se debe a que la reducción de rigideces que resulta de la acción de ciclos alternantes de deformación implica una reducción en la capacidad de disipar energía mediante comportamiento inelástico. Para edificios con características diferentes en cuanto a la capacidad de deformación dúctil, las funciones de confiabilidad que se obtienen son similares, después de cambiar la escala horizontal, de manera de reflejar las diferencias en las capacidades de deformación. De estos estudios se concluye que los criterios de diseño sísmico actuales conducen a niveles de confiabilidad muy variables, aun para estructuras del mismo tipo. Un reto más en el desarrollo de criterios de diseño sísmico basados en desempeño será el de lograr niveles congruentes de confiabilidad entre sistemas estructurales de diversos tipos y propiedades.

Contando con funciones de confiabilidad como las presentadas en la Fig.7, será posible incorporarlas en estudios de optimización similares a los descritos en la Sección 7 de este trabajo. De dichos estudios deberán resultar valores óptimos de las resistencias laterales de estructuras en función 
de sus capacidades de deformación dúctil y de su sensibilidad al deterioro de sus propiedades mecánicas por la acción de cargas repetidas.

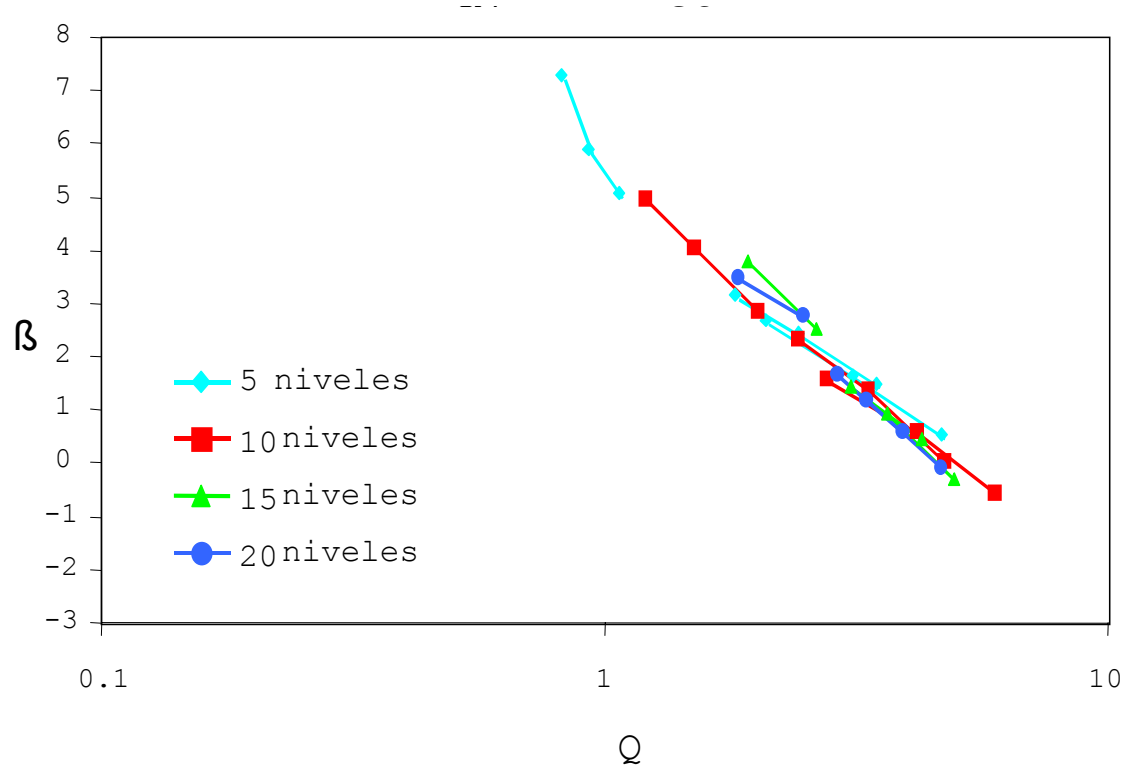

a) Bilineal

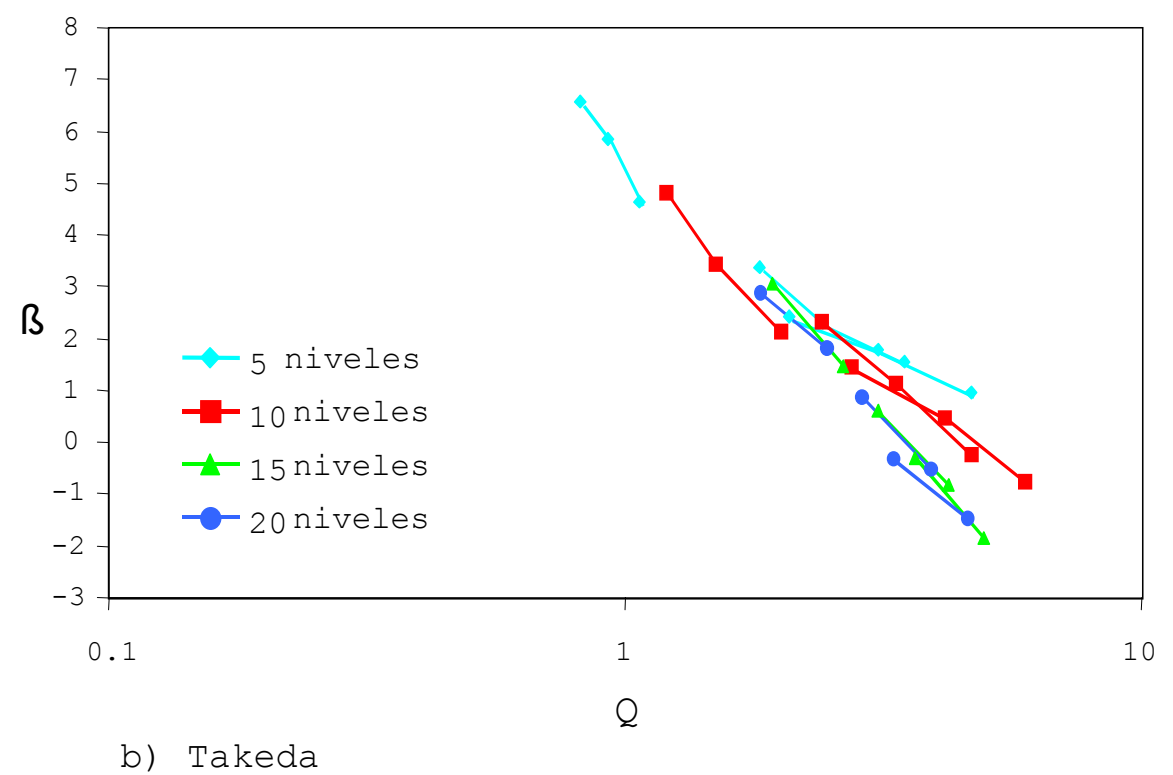

Fig. 5 Funciones de confiabilidad $\beta$ (Q) para marcos de concreto reforzado 


\section{FORMATOS DE DISEÑO SÍSMICO: VARIABLES DE CONTROL Y CRITERIOS DE ACEPTACIÓN}

De acuerdo con lo anterior, la determinación de los valores óptimos de las propiedades mecánicas de un sistema expuesto a las acciones sísmicas puede plantearse en dos formas alternativas, de acuerdo con los parámetros que se usen como variables independientes para fines de minimizar la función objetivo. Según una de ellas, las variables de control serán directamente las propiedades mecánicas de los miembros estructurales (o, más precisamente, las propiedades globales y locales del sistema). Según la otra, tales propiedades se controlan indirectamente mediante los requisitos de resistencia y las limitaciones que se fijen a las deformaciones ante temblores asociados con intervalos de recurrencia especificados. En atención a las herramientas disponibles en condiciones prácticas, conviene que las normas de diseño se expresen en términos de requisitos de resistencia y de cotas superiores a las deformaciones para temblores de distintas intensidades, independientemente de las variables que se hayan empleado en los estudios de optimización.

Dado que las variables de control serán en general las distorsiones laterales (de entrepiso), y que por razones de simplicidad tales distorsiones se estimarán con base en el sistema simplificado equivalente descrito en la Sección 5 de este trabajo (Estimación de Respuestas Sísmicas), convendrá caracterizar a los temblores de diseño en términos de sus espectros de respuesta no lineales (de aceleraciones o de desplazamientos) o de capacidad (Chopra y Goel, 1999; Esteva et al, 1999) correspondientes a diversos niveles de ductilidad. También por razones de simplicidad, los espectros no lineales que se especifiquen corresponderán en general a sistemas histeréticos de tipo bilineal, con pendiente de post-fluencia nula o pequeña, y no considerarán explícitamente los efectos de degradación de rigidez y resistencia asociados a ciclos repetidos de deformación. Habrá que complementar los espectros de diseño con factores que corrijan las distorsiones laterales para tomar en cuenta estos efectos, así como las incertidumbres asociadas con las relaciones entre las distorsiones laterales que se calcularían con modelos detallados del sistema y las que resultarían de aplicar el sistema simplificado equivalente. Los factores correctivos en cuestión también deberían cubrir las diferencias que provienen de calcular las respuestas del sistema con base en sus propiedades esperadas (más probables) que las que resultarían de considerar los valores nominales supuestos en el diseño. La determinación de estos factores es un asunto que requiere atención a corto plazo.

\section{COMENTARIOS FINALES}

Se ha tratado de mostrar un panorama de las tendencias en el desarrollo de normas de diseño sísmico capaces de conducir a niveles congruentes de desempeño y confiabilidad. Para los diseñadores, los nuevos criterios implicarán un control más directo de las variables significativas que afectan el comportamiento esperado de las construcciones para sismos de diversas intensidades. Para los responsables de formular normas y recomendaciones para diseño, el desarrollo de normas y recomendaciones implicará un serio desafío: por una parte, determinar valores óptimos de las propiedades de diversos tipos de sistemas, dentro de un marco de referencia que incluye las acciones probables que puedan experimentar durante su ciclo de vida $\mathrm{y}$, por otra, formular criterios de diseño capaces de producir sistemas que posean dichas propiedades óptimas; todo esto, manteniendo formatos de diseño suficientemente simples para ser aplicados con éxito en condiciones prácticas. 


\section{RECONOCIMIENTO}

El autor agradece la revisión crítica y los valiosos comentarios de la Dra. Sonia E. Ruiz.

\section{REFERENCIAS}

Chopra, A.K., y Goel, R.K., "Capacity-demand-diagram methods for estimating seismic deformations of inelastic structures: SDF systems”, Report No. PEER-1999/02, Pacific Earthquake Engineering Research Center, University of California, Berkeley (1999).

Cornell, C.A., "A probability-based structural code", Journal of the American Concrete Institute, 66, 12 (1969).

Esteva, L. y Ruiz, S.E., "Seismic failure rates of multistory frames", ASCE Journal of Structural Engineering, 115, 2 (1989) 268-284.

Esteva, L., Ruiz, S.E., Veras, L. y Díaz, V., "Reliability and performance-based seismic design criteria for structures with energy-dissipating devices: present experience and trends in Mexico", Proc., International Post-SMIRT Conference Seminar on Seismic Isolation, Passive Energy Dissipation and Active Control of Vibrations of Structures, Cheju, Corea (1999).

Esteva, L., Díaz, O., García, J., Sierra, G. e Ismael, E., “Bases para establecer normas de diseño sísmico con criterios de desempeño para construcciones en el Valle de México", informe del Instituto de Ingeniería al Gobierno del Distrito Federal (2000).

Esteva, L., Díaz, O. y García, J., "Reliability functions for earthquake resistant design", enviado para publicación en un volumen especial de Reliability Engineering and System Safety (2000a).

Esteva, L., Díaz-López, O., García-Pérez, J., Sierra, G. e Ismael, E., "Life-cycle optimization in the establishment of performance-acceptance parameters for seismic design", artículo enviado para publicación en las memorias de ICOSSAR-2001 (Congreso Internacional sobre Seguridad y Confiabilidad Estructural), Newport Beach, California (2001).

Otani,S., "Inelastic analysis of R/C frame structures", ASCE Journal of the Structural Division, 100, ST7 (1974), 1433-1449.

Qi, X. y Moehle, J.P., “Displacement Design Approach for Reinforced Concrete Structures Subjected to Earthquakes”, Report No. UCB/EERC-91/02, University of California at Berkeley (1991).

Reyes, C., "El estado límite de servicio en el diseño sísmico de edificios", Tesis Doctoral, Facultad de Ingeniería, UNAM (1999).

Rosenblueth, E., "Optimum design for infrequent disturbances", ASCE Journal of the Structural Division, 102, ST9 (1976), 1807-1825.

SEAOC, Structural Engineers Association of California, Performance Based Seismic Engineering of Buildings, informe final del Comité VISION 2000, Vols. 1 y 2 (1995).

Vargas, E., "Influencia del coeficiente sísmico de diseño en el costo de edificios con marcos de concreto", Tesis de Licenciatura, Escuela Superior de Ingeniería y Arquitectura, IPN (1991). 


\section{APÉNDICE. Estimación de respuestas sísmicas de sistemas no lineales de múltiples grados de libertad por medio de sistemas simplificados equivalentes}

Considérese un sistema de múltiples grados de libertad con las siguientes propiedades:

$\boldsymbol{M}=$ matriz de masas

$\boldsymbol{K}=$ matriz de rigideces

$\Phi=$ configuración deformada en el análisis de empuje lateral; esta configuración se considera igual a la configuración dominante durante la respuesta sísmica

$V=$ fuerza cortante en la base

$u_{N}=$ desplazamiento del extremo superior, relativo a la base

$V_{y}, u_{N y}=$ fuerza cortante en la base y desplazamiento del extremo superior, ambos en el instante en que ocurre la fluencia en la curva $V$ - $u_{N}$

De igual manera, las propiedades del sistema simplificado equivalente son las siguientes:

$m=$ masa

$k=$ rigidez lateral

$u=$ desplazamiento relativo a la base

$v=$ fuerza cortante

$v_{y}, u_{y}=$ fuerza cortante y desplazamiento de fluencia

Además, $\zeta$ es la fracción del amortiguamiento crítico en ambos sistemas.

Supóngase ahora que la configuración del modelo detallado del sistema se mantiene igual a $\Phi$ durante toda la historia de su respuesta sísmica a una aceleración de la base igual a $\ddot{x}_{0}(t)$. Bajo esta hipótesis, la ecuación de movimiento es la siguiente:

$$
m_{1} u_{N}+2 \zeta p m_{1} u_{N}+k_{1} g\left(u_{N}, u_{N}\right)=-r m_{1} \ddot{x}_{0}
$$

En esta ecuación,

$$
\begin{aligned}
& p=k_{1} / m_{1}, \quad k_{1}=\Phi^{T} \boldsymbol{K} \Phi, \quad m_{1}=\Phi^{T} \boldsymbol{M} \Phi \\
& \gamma=\frac{\Phi^{T} \boldsymbol{M} \boldsymbol{J}}{m_{1}}, \quad g\left(u_{N}, \dot{u}_{N}\right)=\text { función de respuesta no lineal }
\end{aligned}
$$

$\boldsymbol{J}$ es un vector formado por componentes iguales a la unidad

Además, se define la variable $r=\Phi^{T} K J / k_{1}$, que se empleará en lo que sigue.

Para el sistema simplificado equivalente, la ecuación de movimiento es la siguiente: 


$$
m \ddot{u}+2 \zeta p m u \dot{u}+k g(u, \dot{u})=-m \ddot{x_{0}}
$$

De lo anterior se tiene que la fuerza cortante en la base del sistema cuando el comportamiento del sistema se encuentra en el intervalo lineal está dada por $V=\boldsymbol{J}^{T} \boldsymbol{K} \Phi u_{N}=\Phi^{T} \boldsymbol{K} \boldsymbol{J}_{N}=r k_{1} u_{N}$ para el modelo detallado, y por $v=k u$ para el sistema equivalente.

De acuerdo con lo anterior, la relación entre las respuestas de ambos sistemas está dada por $u_{N}=\gamma u$. Para que las deformaciones de fluencia se alcancen simultáneamente en ambos sistemas deberá tenerse $u_{N_{Y}}=\gamma u_{y}$. En consecuencia, la relación entre las fuerzas de cortante de fluencia será $v_{y}=k V_{y} /\left(k_{1} r \gamma\right)$. Estas relaciones deben tenerse en cuenta al seleccionar los valores de $k \mathrm{y} v_{y}$ para el sistema simplificado. Para ello se tienen distintas opciones. La más simple es hacer $k=k_{1} r \gamma$, lo que conduce a $v_{y}=V_{y}$. Por otra parte, $m$ deberá hacerse igual a $m_{1} r \gamma$, a fin de conservar el periodo natural. Otra opción es tomar $k=k_{1}$, lo que conduce a $v_{y}=V_{y} /(r \gamma)$ y $m=m_{1}$. En estas condiciones, si se aplica al modelo detallado la excitación $\gamma \ddot{X}_{0}$, resultará que $u_{N}=u, u_{N y}=u_{y}, \mathrm{y} v_{y}=k V_{y} /\left(k_{1} r\right)$. 